\title{
SELETIVIDADE $\pi$-FACIAL EM BIORREDUÇÕES DE NORBORNAN- 7-ONAS 2-ENDO-SUBSTITUÍDAS POR BEAUVERIA DENSA (CMC 3240) E CURVULARIA LUNATA (NRRL 2380)
}

\author{
$\pi$-FACE SELECTIVITY FROM BIOREDUCTIONS OF 2-eNDO-SUBSTITUTED NORBORNAN-7-ones bY \\ BEAUVERIA DENSA (CMC 3240) AND CURVULARIA LUNATA (NRRL 2380)
}

\section{Ana Lúcia Alves de Arruda ${ }^{1}$, Maria Rita Marques ${ }^{2}$, Dênis Pires de Lima ${ }^{3^{*}}$ e Adilson Beatriz $^{4}$}

\author{
${ }^{1}$ Prof ${ }^{\mathrm{a}}$. da Universidade Católica Dom Bosco - UCDB, Campo Grande - MS \\ ${ }^{2}$ Prof $^{\text {a }}$. Adjunta do Departamento de Morfofisiologia - Universidade Federal de Mato Grosso do Sul - UFMS \\ ${ }^{3}$ Prof. Adjunto do Departamento de Química - Universidade Federal de Mato Grosso do Sul - UFMS \\ ${ }^{4}$ Prof. Doutor da Faculdade Estácio de Sá de Campo Grande - FESCG
}

*Autor para correspondência: e-mail: dlima@nin.ufms.br

RESUMO: A redução estereosseletiva dos monoterpenos sintéticos 2-ciano-norbornan-7-ona e 2-p-nitrofenilnorbornan-7-ona por Beauveria densa (CMC 3240) e Curvularia lunata (NRRL 2380) é descrita neste manuscrito. Os microrganismos foram capazes de reduzir as cetonas aos respectivos álcoois isoméricos $(E)$ e $(Z)$. Uma seletividade $\pi$-facial nas biorreduções foi observada e comparada com as seletividades obtidas usando reagentes redutores clássicos.

PALAVRAS-CHAVE: Norbornan-7-onas, biorreduções, seletividade $\pi$-facial, Beauveria densa, Curvularia lunata

ABSTRACT: Selective reduction of synthetic monoterpenes 2-cyano-norbornan-7-one and 2-p-nitrophenylnorbornan-7-one by Beauveria densa (CMC 3240) and Curvularia lunata (NRRL 2380) is reported. The microorganisms were capable of reducing the ketones to the respective $(E)$ and $(Z)$ alcohols. $A \pi$-face selectivity in the bioreductions was observed and compared to found selectivity using classical reducing agents.

KEYWORDS: Norbornan-7-ones, bioreductions, $\pi$-face selectivity, Beauveria densa, Curvularia lunata

\section{INTRODUÇÃO}

O uso de biocatálise como alternativa para reduções assimétricas de cetonas e aldeídos depende da disponibilidade de uma enzima específica ou células microbianas integrais. A última forma é geralmente a preferida para escala industrial, devido aos baixos custos e por evitar o problema de regeneração de co-fatores (SALVI \& CHATTOPADHYAY, 2001; ISHIGE et al, 2005). 
A redução assimétrica de cetonas e aldeídos quirais pode ser feita usando desidrogenases isoladas ou células microbianas integrais, fornecendo os correspondentes álcoois secundários ou primários, respectivamente. Para cetonas, a estereoquímica resultante da reação pode ser prevista com boa exatidão - é determinada principalmente pela natureza da enzima e pelas exigências estereoeletrônicas do composto. Esta racionalização é geralmente conhecida como regra de Prelog (SALVI \& CHATTOPADHYAY, 2001). Para ter acesso a ambos os produtos isoméricos, a disponibilidade de desidrogenases que mostram estereosseletividade opostas nessas biorreduções - isto é, especificidade Prelog (que fornece S-álcoois) e anti-Prelog (que fornece R-álcoois) - é requerida. A maioria das desidrogenases encontra-se definitivamente em favor de enzimas Prelog, enquanto que candidatos complementares são mais difíceis de obter (FABER, 1997).

A seleção facial em adições ao grupo carbonila de norbornanonas endo-substituídas têm recebido muita atenção (MEHTA \& CHANDRASEKHAR, 1999) e a adição nucleofílica à cetonas estericamente impedidas estão ainda sendo bastante investigadas (PRIYAKUMAR et al, 2004). No entanto, não existe relato na literatura sobre modificações dessas moléculas por sistemas biológicos.

Neste contexto, resolvemos realizar uma triagem com vários microrganismos, tais como Cercospora sojina, Cladosporium sphaerospermum, Rhizopus sp., Mucor ramosissimus, Beauveria bassiana, Beauveria densa e Curvularia lunata com a finalidade de obter uma redução seletiva do grupo carbonila 2-ciano-norbornan-7-ona (1) e 2-p-nitrofenil-norbornan-7-ona (2), nas suas formas racêmicas.

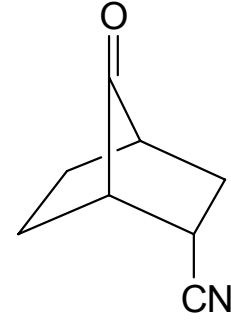

rac-1<smiles>O=C1C2CCC1C(c1ccc([N+](=O)[O-])cc1)C2</smiles>

Figura 1: Estruturas de 2-ciano-norbornan-7-ona (1) e 2-p-nitrofenil-norbornan-7-ona (2).

Entre as cepas de microrganismos testadas, dirigimos nossa atenção aos fungos Beauveria densa (CMC 3240) e Curvularia lunata (NRRL 2380), tendo em vista o fato da existência de trabalhos anteriores com estes microrganismos (GROGAN \& WILLETTS, 1997; DEHLI \& GOTOR, 2001; GONÇALVES, 2002) e também pelo sucesso que tivemos com essas cepas, em estudos de redução e oxidação de sesquiterpenos (LIMA et al, 1999).

Neste trabalho, estamos relatando uma redução diastereosseletiva das cetonas 1 e 2 aos seus álcoois correspondentes, usando B. densa (CMC 3240), e C. lunata (NRRL 2380), respectivamente.

\section{MATERIAL E MÉTODOS}

Reagentes e Microrganismos: 2-p-Nitrofenil-norbornan-7-ona (1) e 2-ciano-norbornan-7-ona (2) foram sintetizadas pelo grupo do Prof. G. Mehta (Escola de Química, Universidade de Hyderabad, Hyderabad, Índia) e doados pelos Prof. S. M. Roberts e A. J. Carnell (Universidade de Liverpool, Reino Unido). B. densa e C. lunata também foram uma doação dos laboratórios do Departamento de Química, Universidade de Liverpool.

Procedimentos Gerais para as Biotransformações: Os microrganismos foram pré-cultivados a $28^{\circ} \mathrm{C}$ e com rotação orbital de $120 \mathrm{rpm}$, em um erlenmmeyer de $250 \mathrm{~mL}$ contendo $100 \mathrm{~mL}$ do meio a seguir: $7,5 \%$ extrato de levedura, $10 \%$ glicose e $1 \mathrm{~L}$ de água destilada em pH 4,85. Após 96 horas, $100 \mathrm{mg}$ de substrato dissolvido em $0,5 \mathrm{~mL}$ de $\mathrm{EtOH}$ foram adicionados nos meios de cultura, amostras foram retiradas do meio e analisadas por CCD. Após detecção do produto (7 dias), o meio de cultura e os micélios foram separados por filtração e o produto foi extraído com EtOAc do filtrado. Maior tempo de incubação não levou a nenhuma outra transformação detectável (por CCD) dos substratos. O solvente foi evaporado e o extrato bruto foi analisado pelo espectro de RMN de ${ }^{1} \mathrm{H} \mathrm{e}$ separados por cromatografia de sílica gel do tipo flash (Merck, 40-63 $\mu \mathrm{m}$ ) usando-se hexano:acetato de etila em um apropriado gradiente de concentração. 
Análises Espectroscópicas e Cromatográficas (CG): A amostra foi dissolvida em acetato de etila (10 $\mathrm{mg} / \mathrm{mL}$ ) e analisada por CG/FID com uma coluna capilar de sílica fundida $(30 \mathrm{~m}, 0,25 \mathrm{~mm}, 1 \mu \mathrm{m}) \mathrm{DB}-5, T_{\mathrm{i}}=80^{\circ} \mathrm{C}$, $T_{\Gamma}=280{ }^{\circ} \mathrm{C}, T_{\text {inj }}=280^{\circ} \mathrm{C}, T_{\text {detect }}=280^{\circ} \mathrm{C}$.

Os espectros de RMN de ${ }^{1} \mathrm{H}$ e de ${ }^{13} \mathrm{C}$ foram obtidos a 300 e $75 \mathrm{MHz}$, respectivamente (Brucker DPX 300). Os deslocamentos químicos foram medidos em ppm tendo como referência interna o TMS. As constantes de acoplamento $(J)$ estão em $\mathrm{Hz}$ (hertz). Espectro de Infravermelho foram determinados em pastilhas de $\mathrm{NaCl}$ ou filme.

Caracterização dos Compostos: 7-Hidróxi-biciclo[2.2.1]heptano-2-carbonitrila, (1a) e (1b): (CG/FID) tempos de retenção $=16,20(21 \%)$ e 16,38 (67\%), respectivamente. $v\left(\mathrm{CH}_{2} \mathrm{Cl}_{2}\right) / \mathrm{cm}^{-1} 3418,2963,2880,2238(\mathrm{CN})$. ${ }^{1} \mathrm{H}$ RMN $\delta 4,15(\mathrm{~s}), 3,89(\mathrm{~s}), 3,21-3.17(\mathrm{~m}), 2,80-2,75(\mathrm{~m}), 2,29-1,86(\mathrm{~m}), 1,48-0,90(\mathrm{~m}) ;{ }^{13} \mathrm{C}$ RMN: $\delta 125,81(\mathrm{CN})$, 121,89 (CN), 79,12, 78,29, 43,84, 43,30, 40,51, 39,96, 32,61, 32,25, 27,69, 27,14, 26,20, 26,11, 22,11, $22,05$.

2-(4-Nitrofenil)-biciclo[2.2.1]heptan-7-ol, (2a) e (2b). Os dados de RMN de ${ }^{1} \mathrm{H}$ de ${ }^{13} \mathrm{C}$ estão de acordo com os reportados na literatura. A proporção entre os isômeros foi determinada a partir das integrações relativas dos prótons metínicos.

\section{RESULTADOS E DISCUSSÃO}

As cetonas $( \pm)$ 2-ciano-norbornan-7-ona (1) e $( \pm)$ 2-p-nitrofenil-norbornan-7-ona (2) foram submetidas à transformação microbiana usando $B$. densa e $C$. lunata e foram produzidas duas misturas diastereoisoméricas de álcoois $(E)$ e $(Z)$.

No caso do composto 1 foi obtida uma mistura dos diastereoisômeros 1a e 1b. Para o composto 2, obtevese a mistura dos isômeros $\mathbf{2} \mathbf{a}$ e $\mathbf{2 b}$.

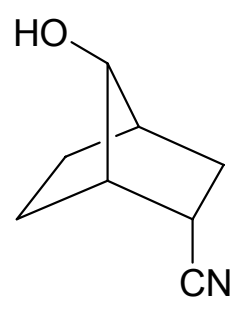

(E) rac-1a



(Z) rac-1b
(E)<smiles>CC(C)(C)Oc1ccc(C2CC3CCC2C3O)cc1</smiles>

(Z)<smiles>O=[N+]([O-])c1ccc(C2CC3CCC2C3O)cc1</smiles>

Figura 2: Estruturas químicas dos norbornanóis obtidos por biorreduções.

Durante o período das biotransformações não foi detectada, através de cromatografia de camada delgada, a formação de nenhum outro produto. A Tabela 1 mostra uma comparação entre os resultados das biotransformações de 1 e 2 com os resultados de reações de redução com boroidreto de sódio reportados na literatura (MEHTA et al, 1992; 1996).

Tabela 1: Biorreduções de Norbonan-7-onas por Beauveria densa (CMC 3240) e Curvularia lunata (NRRL 2380) e redução com $\mathrm{NaBH}_{4}$

\begin{tabular}{ccccc}
\hline Agente redutor & Substrato & $\begin{array}{c}\text { Álcoois [proporção } \\
(E):(Z)]^{\mathrm{a}}\end{array}$ & Rendimento (\%) & Tempo \\
\hline B. densa & $\mathbf{1}$ & $\mathbf{1 a : 1 b}(3: 1)$ & $80^{\mathrm{b}}$ & 15 dias \\
C. lunata & $\mathbf{2}$ & $\mathbf{2 a : 2 b ( 2 : 1 )}$ & $70^{\mathrm{b}}$ & 15 dias \\
$\mathrm{NaBH}_{4}$ & $\mathbf{1}$ & $\mathbf{1 a : 1 b}(4: 1)$ & 90 & - \\
$\mathrm{NaBH}_{4}$ & $\mathbf{2}$ & $\mathbf{2 a : 2 b}(3: 2)$ & 90 & -
\end{tabular}

a A proporção entre os produtos foi determinada pela integração dos sinais de RMN de ${ }^{1} \mathrm{H}$ nos respectivos espectros das misturas e por análise de cromatogramas de CG. 
${ }^{\mathrm{b}}$ Calculado baseando-se no material de partida que efetivamente foi biotransformado.

A estereoquímica dos 7-norbornanóis foi determinada inequivocamente através do espectro de RMN de ${ }^{1} \mathrm{H}$, baseando-se na grande desblindagem relativa dos hidrogênios carbinólicos (H-7, Figura 3) em $\mathbf{1 b}$ e $\mathbf{2 b}$ (Z-álcoois) quando comparados com os hidrogênios correspondentes nos compostos 1a e 2a (E-álcoois), os quais possuem suficiente caracterização na literatura (MEHTA \& KHAN, 1990; MEHTA \& PRAVEN, 1992; GANGULY et al, 1993; MEHTA et al, 1994).

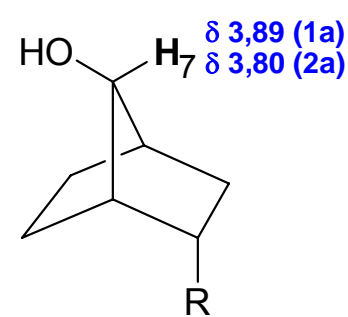

$1 \mathrm{a}, \mathrm{R}=-\mathrm{CN}$

2a, $\mathrm{R}=-\mathrm{C}_{6} \mathrm{H}_{4} \mathrm{NO}_{2}$

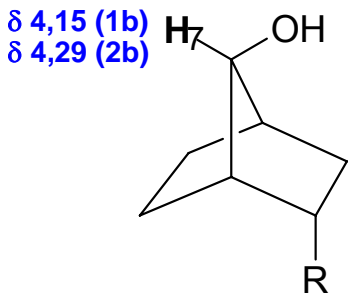

1b, $\mathrm{R}=-\mathrm{CN}$

2b, $\mathrm{R}=-\mathrm{C}_{6} \mathrm{H}_{4} \mathrm{NO}_{2}$

Figura 3: Deslocamentos químicos (ppm) dos hidrogênios carbinólicos observados nos espectros de RMN de ${ }^{1} \mathrm{H}$ dos 7-norbornanóis 1a, 2a, $\mathbf{1 b}$ e $\mathbf{2 b}$.

Os resultados experimentais mostraram uma seletividade $\pi$-facial em ambas as reduções. Estudos deste tipo de seletividade (MEHTA et al. 1992; 1996) em adições nucleofílicas nos compostos 1 e 2 , foram feitos utilizando-se boroidreto de sódio como o agente transferidor de íon hidreto. A adição nucleofílica em norbornan-7onas que possuem grupos eletrocaptores (EWG), acontece preferencialmente de maneira sin, ou seja, do mesmo lado do grupo eletrocaptor, produzindo o álcool $(E)$ como o principal isômero, como mostrado na Figura 4.

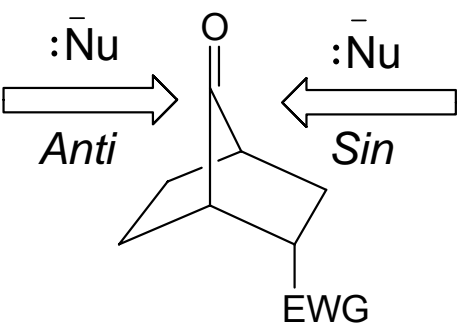<smiles>CC(C)(C)[C@H]1C[C@@H]2CC[C@H](C2)[C@]1(N)O</smiles>

(E)-álcool - principal<smiles>CC(C)C1CC2CCC1C2(C)O</smiles>

(Z)-álcool

Figura 4: Representação esquemática de adição nucleofílica ao grupo carbonila de norbornan-7-onas 2-endosubstituídas.

MEHTA e colaboradores (1992; 1996) racionalizaram esta estereosseletividade baseados no fato de que a discriminação $\pi$-facial é predominantemente determinada pelas interações eletrostáticas e de orbitais existentes entre o nucleófilo, que se aproxima do grupo carbonila, com o grupo eletrocaptor ( $p$-nitrofenil ou ciano, neste caso). O grupo eletrocaptor exerce um efeito estereoeletrônico de longo alcance que influencia na seletividade da face do grupo carbonila.

A seletividade observada pra o composto 1 não foi muito expressiva; no entanto, para a cetona 2 a estereosseletividade foi notadamente mais significativa quando comparada com as reduções efetuadas com $\mathrm{NaBH}_{4}$ (Tabela 1).

Uma provável sugestão para a explicação de adição de íons hidreto nas duas faces dos substratos 1 e 2, seria a presença de múltiplas oxidorredutases, de configurações opostas, presentes na maquinaria enzimática destes microrganismos, os quais competem entre si pelo mesmo substrato, gerando álcoois de diferentes configurações.

Hipoteticamente, podemos considerar que nestas biorreduções há a participação do co-fator NADH ou NADPH de álcoois desidrogenases (cetorredutases) e, de acordo com a regra de Prelog, o hidreto seria transferido pela face re da carbonila, resultando na formação de S-álcoois, conforme representação esquemática no Esquema 1. 


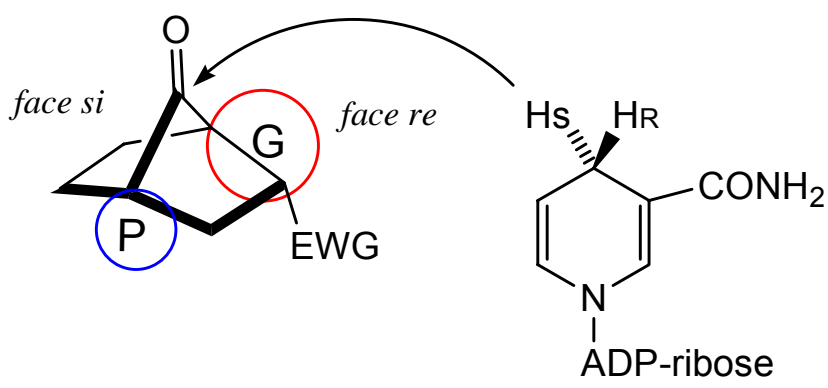

Esquema 1: Estereoquímica da transferência de hidreto por NADH ao carbono da carbonila do substrato $(P=$ Grupo pequeno e $\mathrm{G}=$ Grupo grande).

No caso da mistura racêmica de 1 ou 2, existem duas faces re, como representada pelas estruturas $\mathbf{3}$ e $\mathbf{4}$, no Esquema 2. Então, se estas biorreduções seguirem exclusivamente a regra de Prelog, produziria uma mistura diastereoisomérica de $(E)$ e $(Z)$-álcoois em iguais proporções. A mesma racionalização pode ser feita para o ataque do hidreto pelas duas faces si. Contudo, em nossos experimentos, foram formados predominantemente (E)-álcoois, isto sugere, portanto, que os efeitos eletrostáticos e de orbitais dos grupos EWG $\left(-\mathrm{CN} \mathrm{e}-\mathrm{C}_{6} \mathrm{H}_{4} \mathrm{NO}_{2}\right)$, desempenha um importante papel na estereosseletividade. Baseando-se neste raciocínio, pode-se sugerir que, assim como na redução com boroidreto de sódio, esses grupos são também sin-dirigentes nas reduções promovidas pelos microrganismos testados.

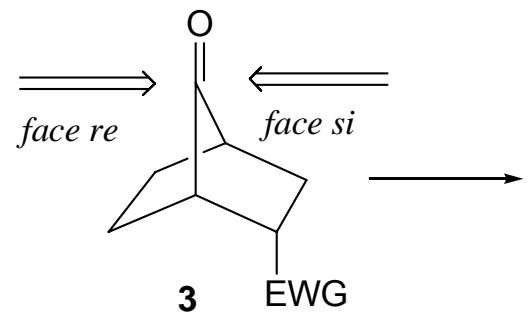<smiles>CC(C)(C)[C@H]1C[C@@H]2CC[C@H](C2)[C@H]1O</smiles>

(E)-álcool anti-Prelog - 3a

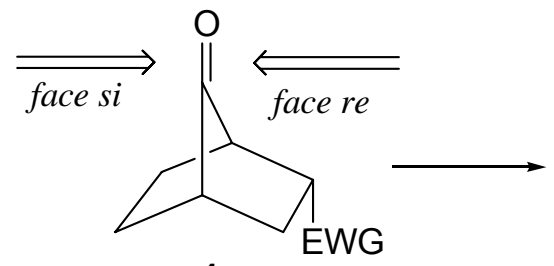

4

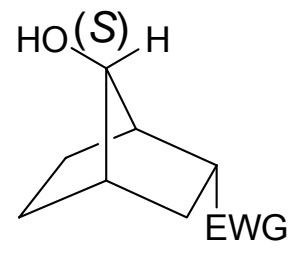

(E)-álcool Prelog - 4a<smiles>CC(C)(C)[C@H]1C[C@@H]2CC[C@H](C2)[C@H]1O</smiles>

(Z)-álcool Prelog - 3b

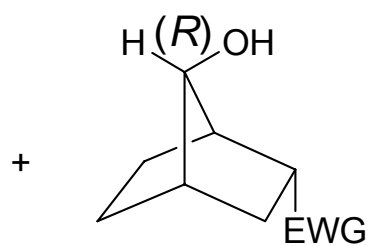

(Z)-álcool anti-Prelog - 4b

Esquema 2: Resultado hipotético de adição nucleofílica Prelog e anti-Prelog do íon hidreto a 2-endo-norbornan-7onas-substituídas

\section{CONCLUSÃO}

Os fungos Beauveria densa (CMC 3240) e Curvularia lunata (NRRL 2380) foram capazes de promover reduções estereosseletivas dos monoterpenos sintéticos 2-ciano-norbornan-7-ona e 2-p-nitrofenil-norbornan-7ona. A hipótese de que os grupos eletrocaptores ou doadores de elétrons têm um impacto nesta estereosseletividade, poderá ser comprovada através da realização de experimentos com desidrogenases isoladas.

\section{AGRADECIMENTOS}

O presente estudo foi financiado pela CAPES e CNPq (Brasil). Os autores agradecem aos professores S. M. Roberts e A. J. Carnell (Universidade de Liverpool, Reino Unido) pelas amostras dos substratos, cepas de microrganismos e por nos encorajar a realizar este trabalho. 


\section{REFERÊNCIAS}

DEHLI, J. R.; GOTOR, V. Stereoselective alkylation-reduction of beta-keto nitriles by the fungus Curvularia lunata. Tetrahedron Asymmetry. v. 12, n. 10, p. 1485-1492, 2001.

FABER, K. Biotransformations in Organic Chemistry. Berlin Heidelberg:Springer-Verlag, 1997.

GANGULY, B. et al. A simple computational model for predicting pi-facial selectivity in reductions of sterically unbiased ketones. Relative importance of electrostatic and orbital interactions. The Journal of Organic Chemistry. v. 58, n. 7 , p. 1734-1739, 1993.

GONÇALVES, R. A. C. Intermediários sintéticos versáteis enantiomericamente puros, obtidos por biocatálise. 2002. Tese (Doutorado) Universidade Estadual de Campinas, Campinas.

GROGAN, G.; RIPPÉ, C.; WILLETTS, A. Biohydrolysis of substituted styrene oxides by Beauveria densa CMC 3240. Journal of Molecular Catalysis B-Enzymatic. V. 3, n. 5, p. 253-257, 1997.

ISHIGE, T.; HONDA, K.; SHIMIZU, S. Whole organism biocatalysis. Current Opinion in Chemical Biology. v. 9, p. 174-180, 2005.

LIMA, D. P. DE; CARNELL, A. J.; ROBERTS, S. M. Microbial Transformation of (+)-10 -14-dihydroxi-alloaromadendrane and (-)-allo-aromadendrone. Journal Chemical Research (S). p. 396-397, 1999.

MEHTA, G.; KHAN, F. A. Electronic control of $\pi$-facial selectivities in nucleophilic additions to 7-norbornanones. Journal of The American Chemical Socity. v. 112, n. 16, p. 6140-6142,1990.

MEHTA, G.; PRAVEN, M. Stereochemistry of nucleophilic additions to 2,3-endo,endo-bridged-7-ketonorbornanes [tricyclo $\left(5.2 .1 .0^{2,6}\right)$ decan-10-ones]. Observation of long-range electronic effects. Tetrahedron Letters. v. 33, n. 13, p. 1759-1762. 1992.

MEHTA, G.; KHAN, F. A.; Lakeshmi, K. A. Modification of m-face selectivity of 7-norbornenones during reduction in $\beta$-cyclodextrin and solid state. Tetrahedron Letters. v. 33, n. 51, p 7977-7980, 1992

MEHTA, G. et al. pi-Facial diastereoselection in reductions of sterically unbiased ketones containing the norbornyl framework - further tests for theoretical-models. Journal of the Chemical Society-Perkin Transactions 2. v. 11, p. $2275-2277,1994$

MEHTA, G. et al. pi-Face selectivities in nucleophilic Additions To 2-Endo-Aryl-Norbornan-7-Ones: The Role Of Through-Space Electrostatic Interactions. Journal of the Chemical Society-Perkin Transactions 1. v. 22, p. 26652667, 1996.

MEHTA, G.; CHANDRASEKHAR, J. Electronic control of facial selection in additions to sterically unbiased ketones and olefins. Chemical Reviews. V. 99, p. 1437-1467, 1999.

PRIYAKUMAR, U. D.; SASTRY, G. N.; MEHTA, G. Development of predictive models of pi-facial selectivity; a critical study of nucleophilic addition to sterically unbiased ketones. Tetrahedron. v. 60, n. 15, p. 3465-3472, 2004.

SALVI, N. A.; CHATTOPADHYAY, S. Studies on Rhizopus arrhizus mediated enantioselective reduction of arylalkanones. Tetrahedron. v. 57, pg. 2833-2839, 2001. 